\title{
Stress
}

The International Journal on the Biology of Stress

\section{Acute stress amplifies experienced and anticipated regret in counterfactual decision- making}

\section{Yin Wu, Eric van Dijk \& Hong Li}

To cite this article: Yin Wu, Eric van Dijk \& Hong Li (2021) Acute stress amplifies experienced and anticipated regret in counterfactual decision-making, Stress, 24:4, 359-369, DOI: 10.1080/10253890.2020.1813275

To link to this article: https://doi.org/10.1080/10253890.2020.1813275

View supplementary material $[\nearrow$

\section{Published online: 08 Sep 2020.}

Submit your article to this journal $[\pi$

Џ Article views: 93

Q View related articles $\complement$

View Crossmark data ¿ 


\title{
Acute stress amplifies experienced and anticipated regret in counterfactual decision-making
}

\author{
Yin $\mathrm{Wu}^{\mathrm{a}, \mathrm{b}}$, Eric van Dijk $\mathrm{c}^{\mathrm{c}}$ and Hong $\mathrm{Li}^{\mathrm{a}, \mathrm{b}}$

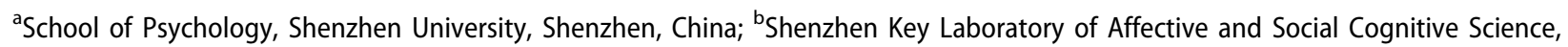 \\ Shenzhen University, Shenzhen, China; 'Department of Social, Economic and Organizational Psychology, Leiden University, Leiden, \\ Netherlands
}

\begin{abstract}
Previous research has shown that stress can affect emotion processing in a variety of settings. However, little attention has been paid to the effects of stress on emotional decision-making. The present study addressed this question by exposing healthy young participants either to a stressor $(n=30)$-socially evaluated cold pressor task- or a non-stressful control task $(n=30)$. Subsequently, participants completed a computerized decision-making task in which they could compare the obtained factual outcome with a non-obtained counterfactual outcome. Saliva samples were taken at four time points over the course of the experiment and used to analyze cortisol levels. Results revealed that acute stress induced reliable salivary cortisol increase over the experimental task. At the outcome delivery stage, acute stress amplified negative emotions induced by the counterfactual comparison. At the choice stage, under stress, participants were more likely to make regret-averse decisions. The findings that acute stress amplifies both experienced and anticipated regret is consistent with dual process frameworks such that stress tilts decision-making toward more emotional and intuitive processing.
\end{abstract}

\section{LAY SUMMARY}

- Stress is thought to affect emotional processing. The present study investigated the effects of acute stress on emotional decision making using a typical counterfactual decision making task. Acute stress amplified both experience and anticipation of regret, consistent with the dual process frame that stress tilts decision-making toward more emotional and intuitive processing.
ARTICLE HISTORY

Received 25 March 2020

Accepted 17 August 2020

\section{KEYWORDS}

stress; cortisol; counterfactual thinking; regret; dual process; emotion

\section{Introduction}

Stress is ubiquitous in our social life. Stress occurs whenever a demand exceeds the regulatory capacity of an organism, particularly in unpredictable and uncontrollable situations (Dickerson \& Kemeny, 2004). Stress induces physiological and endocrine responses, and has a large effect on memory, attention and executive functioning (Roozendaal et al., 2009). Given these pervasive effects, there is ample reason to expect that it will also affect the decisions we make. Many decisions must be made under stress, from choosing correct alternatives in an examination to making important financial decisions at the London Stock Exchange. There is increasing interest in the effects of stress on human decision-making since stress and decision-making are intimately related. Brain regions that are known to underlie decision-making, such as prefrontal, limbic and basal ganglia regions, have numerous receptors for stress hormones (see Starcke \& Brand, 2012 for a review).

Previous research has shown that stress can affect emotion processing in a variety of settings. Neuropsychological research has shown that brain regions associated with the regulation of the acute stress responses are also involved in emotional recognition (Pessoa \& Adolphs, 2010), and the anticipation of social stressors elicited enhanced neural processing for emotional threatening faces (Wieser et al., 2010). These findings are supported in other lines of research. Acute social stress has, for example, been found to facilitate detection of emotional cues, which could help individuals to detect potential threats or sources of social support in the external environment (Domes \& Zimmer, 2019). Stressed individuals also tend to exhibit more emotional empathy (Wolf et al., 2015). Thus, these different lines of research all connect stress to emotional responses.

There is increasing interest in looking at the effects of stress on emotional decision-making. Part of this research focuses on the effects of chronic stress, but there is also increased attention to the effects of acute stress. As for chronic effects, prior research has focused on maladaptive decision-making among individuals with mood and anxiety disorders, which is usually associated with exposure to chronic stress (see Bishop \& Gagne, 2018 for a review). For instance, low-anxious individuals exhibited increased loss aversion under emotional priming, and this emotional 
modulation of loss aversion was associated with a corresponding emotion-induced increase in amygdala-striatal functional connectivity (Charpentier et al., 2016). Moreover, individuals with pathological anxiety demonstrated enhanced risk aversion rather than loss aversion in economic decisionmaking (Charpentier et al., 2017). Patients with major depressive disorder demonstrated hyper-sensitivity to losses in the anterior insula, suggesting abnormal emotional processing (Engelmann et al., 2017).

In addition to these chronic manifestations, research has now also started to document effects of acute stress on emotional decision making. For example, anticipatory stress induced by giving a public speech impaired learning of the reward contingencies in the lowa Gambling Task (IGT), and this effect further depended on the nature of the task and gender of the participants (Preston et al., 2007). Acute stress induced by threat of unpredictable shocks led to contrasting effects on decision-making tendencies depending on mood and anxiety symptoms in the IGT (Robinson et al., 2015). In particular, individuals with high trait anxiety exhibited riskseeking tendencies, while those with low trait anxiety showed risk avoidant tendencies. Repetitive negative thinking (RNT) induced by a natural stressor was associated with greater increases in loss aversion (i.e. overweighing potential monetary losses relative to gains) in decision-making during the early-phase response to a laboratory stressor, highlighting the role of loss aversion and stress in understanding RNT as an affective vulnerability factor (Stamatis et al., 2020).

In the current study, we further explore the effects of acute stress on emotional decision-making by assessing how acute stress impacts the importance of regret. Regret is considered to be one of the main emotional factors underlying decision-making; a feature that is generally attributed to its dependence on counterfactual thinking (Van Dijk \& Zeelenberg, 2005; Zeelenberg, 1999). When making decisions, people often rely on a comparison between obtained decision outcomes, and outcomes that "could have been" (i.e. counterfactual outcomes). The potential connection between acute stress and regret is that stress may impact this process. For example, acute stressors characterized by unpredictability has been hypothesized to elicit a prediction error (i.e. the difference between the obtained and the expected reward) that acts as a teaching signal for the brain and promotes fast learning of the ongoing events (Trapp et al., 2018). Moreover, electrophysiological studies demonstrated that subsecond dopamine fluctuations encode prediction errors associated with processing counterfactual outcomes (Kishida et al., 2016). Thus, it is plausible that stress could affect counterfactual-related decision-making. In the present study, we investigated whether and how acute stress affects the experience of decision outcomes (i.e. does it increase experienced regret?) and how it affects the decisions people make (i.e. does it induce people to make more regret averse decisions?). This distinction relates to the conceptual distinction of experienced and anticipated regret. Regret is an intense negative emotional experience induced by counterfactual comparison, when our current situation ("what is") is worse than an alternative that we could have obtained, had we acted differently ("what could have been"; Mellers et al.,
1999). In a decision-making task, individuals usually feel negative when their actual obtained outcome is worse than the alternative, counterfactual outcome. Regret also has a prospective effect on decision making. In particular, individuals tend to make choices as to minimize the anticipated regret, i.e. the regret that may result from their decisions (Camille et al., 2004; Coricelli et al., 2005). Previous research has shown that brain regions involved in emotional processing, such as orbitofrontal cortex and amygdala, are associated with experienced and anticipated regret (Camille et al., 2004; Coricelli et al., 2007, 2005).

Here we presented half of our participants with an acute stress-evoking task, i.e. the Socially Evaluated Cold Pressor Task (SECPT) (Schwabe et al., 2008; Schwabe \& Schächinger, 2018), while the other half engaged in a non-stressful control task. Subsequently, all participants were presented with a series of trials in which they could choose between two gambles with different probabilities of gain or loss. We manipulated the expected value of the gambles and their potential to induce regret across trials. With this paradigm we were able to assess how stress affects how people experience their decision outcomes as well as how stress affects the decision-making process. Experiences were tapped by having the participants rate their outcomes. Decision-making was analyzed by using a mathematical model in which we also tested the influence of expected value and risk variance (Camille et al., 2004; Gillan et al., 2014). Importantly, we employed a computational modeling approach derived from behavioral economics to uncover the latent decision-making process that may be invisible in simple choice analysis (Gillan et al., 2014; Montague et al., 2012). The advantage of computational modeling is that it allows one to test to what extent behavioral data fit the assumed underlying framework. In the current study, it allowed us to assess the influence of anticipated regret, risk variance and expected value in the stressrelated decision-making process. Given that stress potentiates emotional processing, we hypothesized that acute stress would increase emotional sensitivity to regrettable outcomes (i.e. when obtained outcomes are worse than counterfactual outcomes) and increase the making of regret-aversive decisions (i.e. avoid decisions in which obtained outcomes might be worse than counterfactual outcomes). Following previous published work (Diller et al., 2011; Raio et al., 2017; Speer \& Delgado, 2017), we also administered a series of individual difference measures to make sure the two groups did not differ on traits that are susceptible to acute stressors, including the State-Trait Anxiety Inventory $\left(\mathrm{STAl}{ }^{1}\right.$ (Spielberger et al., 1983), Barratt Impulsiveness Scale ${ }^{2}$ (Barratt et al., 1994) and the Connor-Davidson Resilience $\mathrm{Scale}^{3}$ (Connor \& Davidson, 2003).

\section{Methods}

\subsection{Participants}

Sixty healthy individuals $\left(30\right.$ females $^{4}{ }^{4}$ mean age $=$ 21.92 years, $\mathrm{SD}=2.15$ ) with normal body weight (BMI: $20 \pm 2.45 \mathrm{~kg} / \mathrm{m}^{2}$ ) were recruited through university advertisement. They were randomly assigned to the stress condition 
( $n=30)$ or the control condition $(n=30)$. Participants were screened with a telephone interview and considered ineligible to participate if taking psychotropic medications or having any psychiatric/neurological disorders. Participants were instructed to abstain from alcohol, caffeine intake, and smoking for $24 \mathrm{~h}$ before the testing session. Female participants were tested during the luteal phase of their menstrual cycle, the phase in which women were shown to have the most pronounced cortisol reactivity (Kirschbaum et al., 1999). This study was conducted in accordance with Declaration of Helsinki and was approved by Shenzhen University Medical Research Ethics Committee. Written informed consent was obtained from all participants. Participants were paid 40 Chinese Yuan $(\sim 5.8)$ as a participant fee. They were endowed with 10 Yuan to play the decision-making task. The points that were gained or lost during the task were added or subtracted from this initial endowment and added to the participant fee as a bonus payment.

\subsection{General procedure}

All testing sessions were conducted between 13:00 and 19:00 to control for the diurnal circulation of the stress hormone cortisol. Upon arrival at the laboratory, participants were asked to complete a series of questionnaires on demographic information and personality traits. Participants then completed the SECPT or the control procedure (see below). Immediately before and after completing the stress/control procedure, participants indicated on a visual analogue scale (VAS) their perceived stress level (PSL), and how painful, angry they felt. All rating scales ranged between 0 (not at all) to 100 (extremely).

The decision-making task was administered 15 min after the stress induction (see Figure 1(A)). Over the course of the experiment, four saliva samples were collected to assess cortisol as an index of endocrine stress response. The samples were taken immediately before (T0), and $15 \mathrm{~min}$ (T1), $30 \mathrm{~min}$ (T2) and $45 \mathrm{~min}$ (T3) after completion of the SECPT or control procedure. Saliva samples were collected by using passive drool. At the end of the experiment, saliva samples were frozen and stored at $-80^{\circ} \mathrm{C}$. Samples were assayed in a 3200 QRTAP high-performance liquid chromatography tandem mass spectrometer ( $A B I$, USA) equipped with atmospheric pressure chemical ionization sources (LC-APCI-MS/MS). Cortisol was measured in positive mode as described by Gao et al. (2013). The average intra-assay coefficient of variance was $11.07 \%$, and inter-assay coefficient was $12.67 \%$.

\subsection{Stress induction}

Participants were randomly assigned to the stress $(n=30)$ and control $(n=30)$ conditions. In the stress condition, participants performed the SECPT and were asked to immerse their non-dominant hand in an ice water bath $\left(0-4{ }^{\circ} \mathrm{C}\right)$ for $3 \mathrm{~min}$ while being video-taped and monitored by a research assistant (Schwabe et al., 2008). They were instructed not to communicate and were informed that the research assistant would indicate when the test was over. Participants were allowed to remove their hands from the water bath at any time, but if they did, they were asked to keep looking into the camera until the 3-min test time was over and were instructed that they should re-insert their hand in the water. In the control condition, the participants had to keep their hand in a warm water bath $\left(33-35^{\circ} \mathrm{C}\right)$ for $3 \mathrm{~min}$ while the experimenter was in the room but did not videotape them.

\subsection{Decision-making task}

Participants performed 80 trials of the decision-making task modified from Gillan et al. (2014), which involved monetary wins and losses. On each trial, participants chose between two wheels that each displayed a potential gain and a potential loss, and their respective probability. For example, a wheel could display a potential loss of -210 with $25 \%$ probability and a potential gain of +70 with $75 \%$ probability. Or it could, for example, be +210 with $25 \%$ possibility and -75

(A)

Saliva 1 SECPT/ Saliva 2 Counterfactual task Saliva 3 Counterfactual task Saliva 4 control Part 2

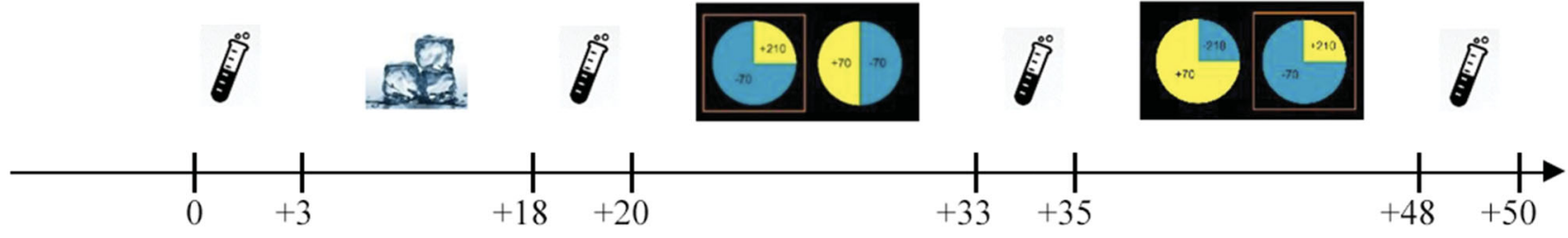

(B)

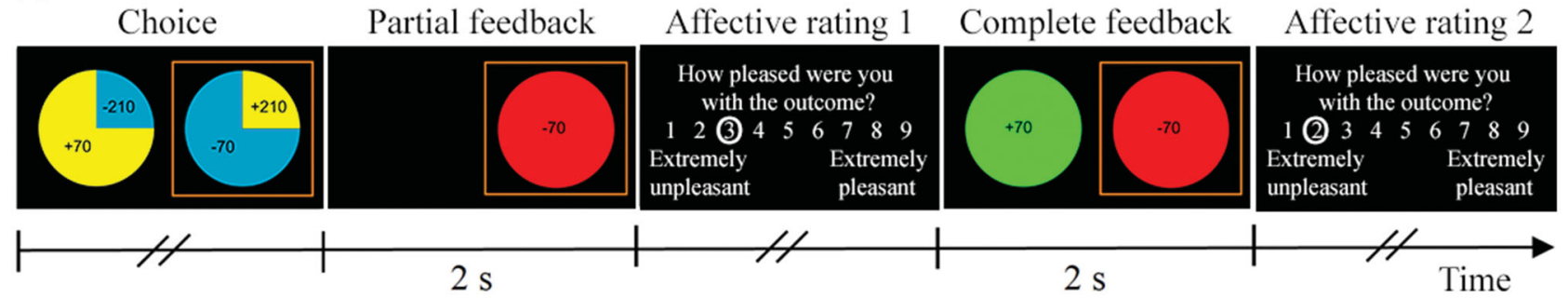

Figure 1. (A) Experimental procedure and timeline. (B) Trial timing for the decision-making task. 
with $75 \%$ possibility. The outcomes and probabilities varied over the trials and between wheels. The positive outcomes were either +70 or +210 points; the negative outcomes were either -70 or -210 points. The outcome probabilities for the two outcomes on a wheel could be $25 \%$ vs. $75 \%$, or $50 \%$ vs. $50 \%$, as indicated by the size of the segment in each wheel (see Figure 1(B)). Participants were informed that each point corresponded to 0.1 Chinese Yuan and these would be added or subtracted from their flat fees.

After the participants selected a wheel, the chosen wheel was highlighted with a red frame. Subsequently, they first only received feedback on the outcome of the chance procedure for their chosen wheel. They thus learned the obtained outcome, as well as the non-obtained outcome of the selected wheel. This information was presented for $2 \mathrm{~s}$, with the non-selected wheel still covered. Participants rated "how pleased were you with the outcome?" using an onscreen rating from 1 (extremely unpleasant) to 9 (extremely pleasant) (henceforth referred to as the Partial Feedback affect rating).

After a $1 \mathrm{~s}$ blank screen, the outcome on the non-selected wheel (i.e. the non-obtained outcome) was presented alongside the obtained outcome for $2 \mathrm{~s}$. Participants again rated how pleased they were with their outcome (henceforth referred to as the Complete Feedback rating). The inter-trial interval was $2 \mathrm{~s}$. No time constraints were imposed on wheel selection or affect ratings. Outcomes were prespecified to be in line with the displayed probabilities, ensuring that the task was fair (see Table S1 of Supplementary Material for the full task sequence).

\subsection{Statistical analysis}

Questionnaire and cortisol data were analyzed using a 2 (treatment) x 4 (time point) mixed analyses of variance (ANOVA) with treatment as the between-participant factor and time point as the within-participant factor. The Bonferroni correction was used for multiple comparisons. The area under curve (AUC) was calculated for the cortisol data. The AUC is a method to aggregate data that assess changes over time, and is frequently used in neuroendocrinological research (Pruessner et al., 2003), with greater AUC values representing stronger cortisol reactivity. Since the AUC values were positively skewed, we log-transformed these values for the statistical analysis.

\subsubsection{Affect ratings}

We used R and Ime4 (Bates et al., 2012) to conduct a linear mixed effect analysis on the affect ratings. All the continuous predictors were transformed into z-scores before statistical analysis. Treatment (stress vs. control, betweenparticipant factor) was entered as a fixed effect factor, trial outcomes (within-participant factor, see below) were continuous fixed effect predictors, and subject was a randomeffect factor. The random effects structure was selected based on the maximal complexity that was supported by our data (Barr et al., 2013).
We conducted separate analyses for the Partial Feedback affect rating and the Complete Feedback affect rating. For the Partial Feedback affect rating, we modeled the influence of (1) the value of obtained outcome, and (2) the chance counterfactual, defined as the difference between the obtained outcome and what could have been obtained in the alternative segment of the chosen wheel. For the Complete Feedback affect rating, we modeled the influence of 1) value of obtained outcome, and (2) the agent counterfactual, defined as the difference between the obtained outcome and the non-obtained outcome on the other wheel (Gillan et al., 2014; Wu et al., 2018).

\subsubsection{Decision making}

We used the model of counterfactual choice from Gillan et al. (2014) to fit participants' wheel selections, to quantify the influence of expected value, risk variance, and anticipated regret in guiding decision-making.

To construct these models, the possible outcomes and associated probabilities were ascribed in the following notations (see Figure 2): $x_{1}$ and $y_{1}$ refer to the two possible outcomes of wheel $1\left(W_{1}\right)$, where $x_{1}>y_{1}$. Similarly, $x_{2}$ and $y_{2}$ refer the two possible outcomes of wheel $2\left(W_{2}\right)$, with $x_{2}>$ $y_{2} . p$ and 1-p are the respective probabilities of earning $x_{1}$ and $y_{1}$ and likewise $q$ and $1-q$ are the respective probabilities of earning $x_{2}$ and $y_{2}$ (see Figure 2). Using this notation, the three decision-making parameters ( $e, v$, and $r$, within-participant factor) were calculated. The parameter that maximizes expected value (EV) is denoted e and is calculated by subtracting the $E V$ of $W_{2}$ from the EV of $W_{1}$. If this value is positive, then a participant seeking to maximize EV should choose $W_{1}$. The $E V$ of $W_{1}$ is calculated using: $\left[p_{1}+(1-p) y_{1}\right]$. The e parameter is thus defined as:

$$
\begin{aligned}
\mathrm{e} & =E \mathrm{~V}_{\mathrm{W} 1}-\mathrm{EV}_{\mathrm{W} 2} \\
& =\left[\mathrm{p} * \mathrm{x}_{1}+(1-\mathrm{p}) * \mathrm{y}_{1}\right]-\left[\mathrm{q} * \mathrm{x}_{2}+(1-\mathrm{q}) * \mathrm{y}_{2}\right]
\end{aligned}
$$

The risk of a wheel can be described as a variance measure capturing the squared difference between each of its outcome and the expected value of the wheel. Higher variance then denotes higher risk. For $W_{1}$ this measure is $p^{*}\left(x_{1}-\right.$ $\left.E V_{W_{1}}\right)^{2}+(1-p)^{*}\left(y_{1}-E V_{W_{1}}\right)^{2}$; for $W_{2}$ this measure is $q^{*}\left(x_{2}-\right.$ $\left.E V_{W_{2}}\right)^{2}+(1-q)^{*}\left(y_{2}-E V_{W_{2}}\right)^{2}$. By comparing these measures, one obtains a measure of the relative risk. The relative risk variance parameter $(\mathrm{v})$ is calculated by subtracting the risk associated with $W_{1}$ from that associated with $W_{2}$. If the value
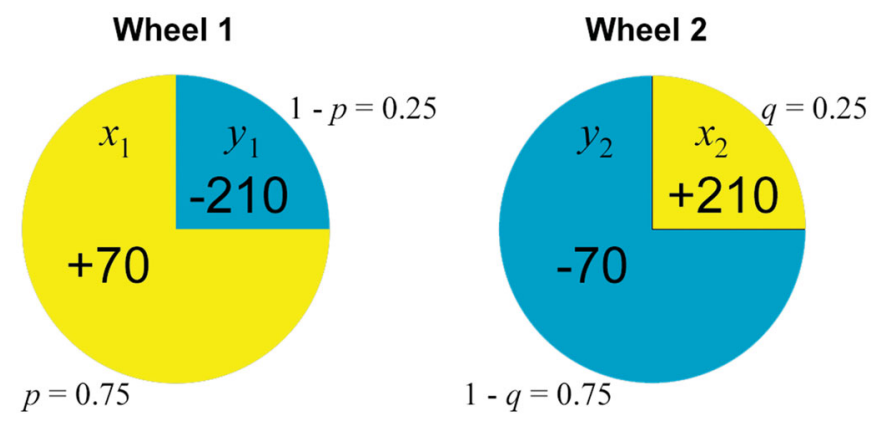

Figure 2. Decision-making model notation. 
is positive, then a participant seeking to minimize or avoid high risk variance should choose $W_{1}$ :

$$
\begin{aligned}
\mathrm{v} & =\mathrm{v}_{\mathrm{w} 2}-\mathrm{V}_{\mathrm{w} 1} \\
& =\left[\mathrm{q} *\left(\mathrm{x} 2-\mathrm{EV}_{\mathrm{W} 2}\right)^{2}+(1-\mathrm{q}) *\left(\mathrm{y}_{2}-\mathrm{EV}_{\mathrm{W} 2}\right)^{2}\right] \\
& -\left[\mathrm{p} *\left(\mathrm{x}_{1}-\mathrm{EV}_{\mathrm{W} 1}\right)^{2}+(1-\mathrm{p}) *\left(\mathrm{y}_{1}-\mathrm{EV}_{\mathrm{W} 1}\right)^{2}\right]
\end{aligned}
$$

Regret would be highest if the outcome of the chosen wheel would be the lowest of its two possible outcomes, and the outcome of the nonchosen wheel would be the highest of its two possible outcomes. The anticipated regret calculation takes into account the magnitude of the difference between the lowest and the highest outcomes across the two wheels. This calculation thereby captures that the greater the difference between obtained and non-obtained outcomes, the greater the experience of regret/relief. Subjects can thus minimize the likelihood of experiencing future regret/relief $(r)$ by making choices associated with the smallest difference in magnitudes, i.e. choosing $W_{1}$ if the outcome of the following equation is positive and choosing $W_{2}$ if it is negative:

$$
r=\left(y_{1}-x_{2}\right)-\left(y_{2}-x_{1}\right)
$$

With the three parameters $v$, e, and $r$, the probability of choosing $\mathrm{W}_{1}\left(\mathrm{Pw}_{\mathrm{it}}\right)$, where $\mathrm{t}$ denotes trial number and $\mathrm{i}$ denotes individual, is calculated using:

$$
P\left(W_{1 i t}\right)=1-P\left(W_{2 i t}\right)=F\left(v_{i t}, r_{i t}, e_{i t}\right)
$$

$F$ is the inverse logit function, $F(\theta)=e^{\theta}\left(1+e^{\theta}\right)$ and $\theta$ is the logit predicted by the individual values of $e, v$ and $r$ in the logistic regression. In keeping with previous studies (Baskin-Sommers et al., 2016; Gillan et al., 2014; Wu et al., 2018), within-subjects logistic regression analysis was performed using the Ime4::Imer function, with the model Choice $\sim \mathrm{e}+\mathrm{v}+\mathrm{r}+$ Treatment:e + Treatment: $\mathrm{v}+$ Treatment: $r$ $+\left(1 \mid\right.$ subject). Choice is a binary variable, coded 1 for wheel $_{1}$ and 0 for wheel $_{2}$. As for the model of affect ratings, treatment was entered as a fixed-effect factor; subject as a random-effect factor; e, v, and $r$ were continuous fixed-effect predictors. Where appropriate, estimated effects from the full model were confirmed with a likelihood ratio test, directly comparing models with and without the term of interest (using the stats::anova function). Logit and inverse logit functions are defined as logit $(\theta)=\ln [\theta /(1-\theta)]$ and invlogit $(\theta)=$ $\mathrm{e}^{\theta}\left(1+\mathrm{e}^{\theta}\right)$, such that invlogit[logit $\left.(\theta)\right]=\theta$.

\subsection{Open Practices}

All the data and analysis scripts are available on the project's Open Science Framework (OSF) page: https://osf.io/hqf7d/.

\section{Results}

\subsection{Participant characteristics}

The two groups did not differ significantly regarding age, BMI, STAI, BIS and CD-RISC (see Table 1).
Table 1. Characteristics of Stress and Control Groups. Individual cells depict means \pm standard deviation of the mean.

\begin{tabular}{lccc}
\hline & $\begin{array}{c}\text { Stress } \\
(n=30, \\
15 \text { males })\end{array}$ & $\begin{array}{c}\text { Control } \\
(n=30, \\
15 \text { males })\end{array}$ & Group comparison \\
\hline Age & $22.7( \pm 2.1)$ & $22.1( \pm 2.4)$ & $t(58)=0.97, p=.337$ \\
BMI & $20.3( \pm 2.6)$ & $19.8( \pm 2.4)$ & $t(58)=0.81, p=.424$ \\
STAI-T & $40.2( \pm 6.4)$ & $37.5( \pm 7.6)$ & $t(58)=1.53, p=.132$ \\
BIS & $64.8( \pm 10.8)$ & $62.2( \pm 7.6)$ & $t(58)=1.09, p=.284$ \\
CD-RISC & $63.7( \pm 12.1)$ & $62.9( \pm 13.0)$ & $t(58)=0.81, p=.806$ \\
\hline
\end{tabular}

\subsection{Stress manipulation}

As expected, the SECPT effectively induced a pronounced subjective and endocrine stress response. Perceived stress level (see Figure $3(\mathrm{~A})$; time $\mathrm{X}$ treatment, $F(1,58)=4.36, p=$ $.041, p \eta^{2}=0.07$ ) and pain (see Figure 3(B); time $\mathrm{X}$ treatment, $F(1,58)=4.41, p=.040, p \eta^{2}=0.071$ ) were higher in the SECPT condition than in the control condition. Treatment did not affect self-reported anger (see Figure $3(C)$; time $X$ treatment, $F(1,58)=1.07, p=.304)$.

The effect of stress manipulation was tested using mixed ANOVA with treatment as the between-participant factor and time point as the within-participant factor. We analyzed salivary cortisol levels as an index of the activity in the HPA axis in response to the SECPT. The interaction between treatment and time was significant, $F(3,174)=12.43, p<.001, p \eta^{2}=$ 0.176 , see Figure 4(A). Further analysis revealed elevated cortisol levels at T1 $(t(58)=5.48, p<.001 \text {, Cohen's } d=1.41)^{5}$, T2 $(t(58)=5.75, p<.001$, Cohen's $d=1.48)$, and T3 ( $t$ (58) $=4.64, p<.001$, Cohen's $d=1.20)$, whereas there was no group difference at T0 $(t(58)=0.83, p=.412)$. The stress condition $(M=11.07, S D=3.57)$ showed a higher total cortisol response (i.e. area under curve) than the control condition $(M=5.89, S D=1.18), t(58)=7.55, p<.001$, Cohen's $d=1.95$, see Figure $4(B)$. Furthermore, participants in the stress condition maintained an increased cortisol level compared to baseline $(t(29)=-2.98, p=.006$, Cohen's $d=0.74$ ) until the end of the behavioral task $(+48 \mathrm{~min})$.

\subsection{Affect ratings with partial feedback}

The partial feedback affect rating model showed a significant main effect of obtained outcome, $b=1.841279$, $S E=0.027967, t=65.84, p<.001$, Cohen's $d=1.92$, with higher pleased rating following more positive obtained outcomes. The main effect of group was not significant. The interaction between obtained outcome and treatment was not significant, $b=0.066838, S E=0.039654, t=1.69, p=.092$ (Figure 5).

There was a significant main effect of chance counterfactual (the difference between the obtained outcome and the alternative outcome on the chosen wheel), $b=1.742666$, $S E=0.03028, t=57.54, p<.001$, Cohen's $d=1.68$, such that pleased ratings were higher when the obtained outcome was greater than the non-obtained, and pleased ratings were lower when the obtained outcome was smaller than the nonobtained. The interaction between treatment and chance counterfactual was significant, $b=0.09292, \quad S E=0.04297$, $t=2.16, p=0.031$, Cohen's $d=0.06$. In the control condition 
(A)

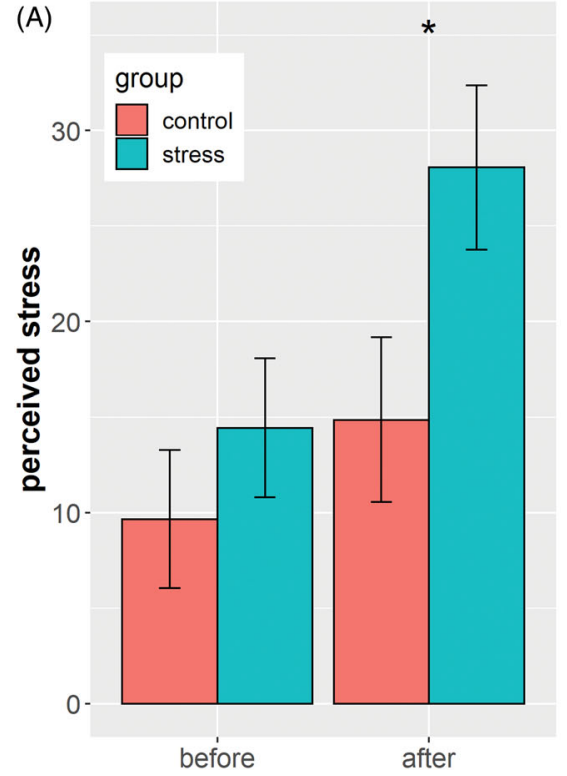

(B)

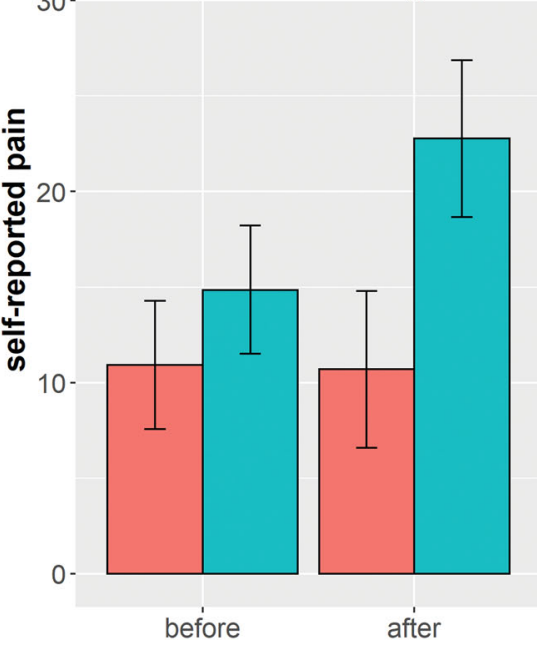

(C)

30

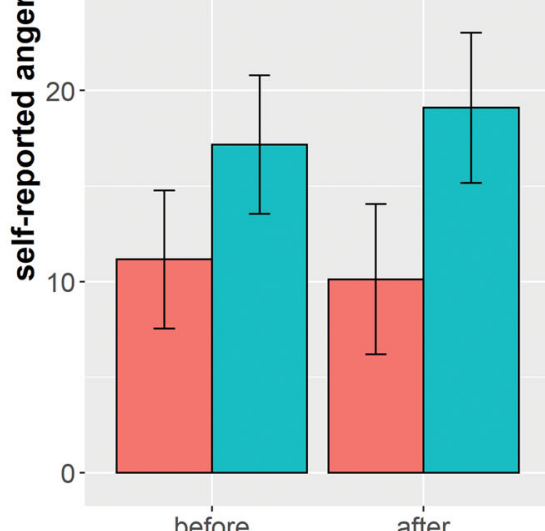

Figure 3. The SECPT procedure increased self-perceived stress (A) and self-reported pain (B) but had no effect on self-reported anger (C). Error bars represent standard error of means.

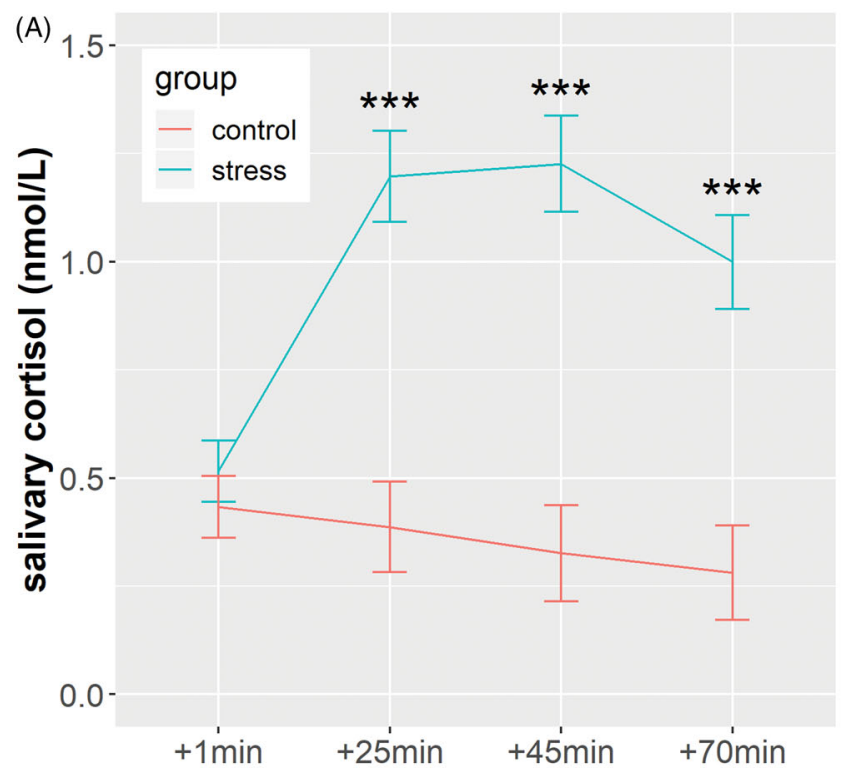

(B)

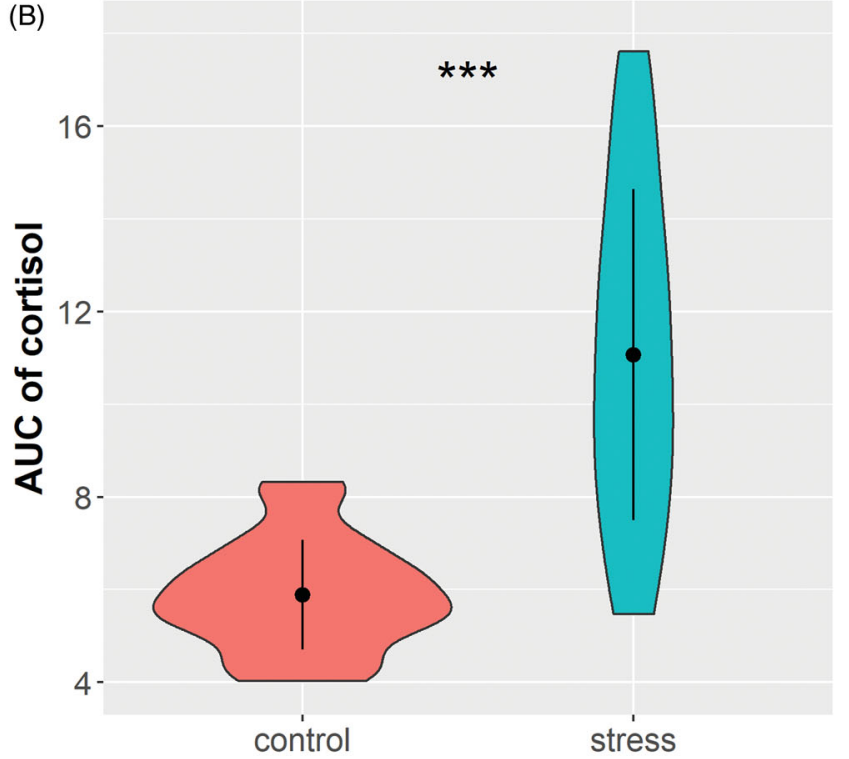

Figure 4. (A) The average salivary cortisol levels for the stress and control group at T0 (immediately before the procedure), T1 (15 min), T2 (30 min) and T3 (45 min). Error bars represent standard error of means. (B) Compared to the control group, the stress group showed greater AUC. Each point within a violin plot represents mean of AUC within each group, the shape represents data distribution. ***refers to $p<.001$.

group, affective ratings increased with the magnitude of chance counterfactual, $b=1.74283, S E=0.03006, t=57.97, p$ $<.001$, Cohen's $d=2.39$. This effect was greater in the stress group, $b=1.83542, \quad S E=0.03071, \quad t=59.77, \quad p<.001$, Cohen's $d=2.46$.

\subsection{Affect ratings with complete feedback}

Similar to the partial feedback analysis, the complete feedback affect rating model (i.e. following the feedback of the non-chosen wheel) showed a significant main effect of obtained outcome, $b=1.51027, S E=0.03681, t=41.03$, $p<.0001$, Cohen's $d=1.20$. The interaction between treatment and obtained outcome was significant, $b=0.13215$,
$S E=0.05219, t=2.53, p=.011$, Cohen's $d=0.07$. Further analysis revealed that the positive association between obtained outcome and the complete feedback affect rating was stronger in the stress condition $(b=1.64242$, $S E=0.03749, t=43.81, p<.001$, Cohen's $d=1.80)$ than in the control condition $(b=1.51027, S E=0.03631, t=41.59$, $p<.001$, Cohen's $d=1.71$ ).

In the model for agent counterfactual (i.e. the difference between the obtained outcome and the non-obtained outcome on the rejected wheel), there was a significant main effect of agent counterfactual, $b=1.59215, S E=0.03501$, $t=45.47, p<.001$, Cohen's $d=1.32$. The interaction between agent counterfactual and treatment was significant $(b=0.17284, \quad S E=0.04960, t=3.48, p<.001$, Cohen's $d=0.10$ ). The positive association between agent 
(A)

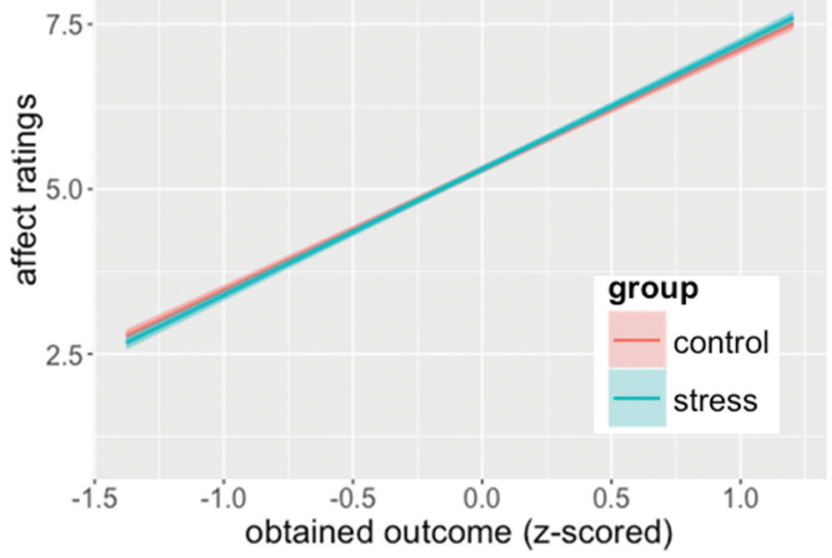

(C)

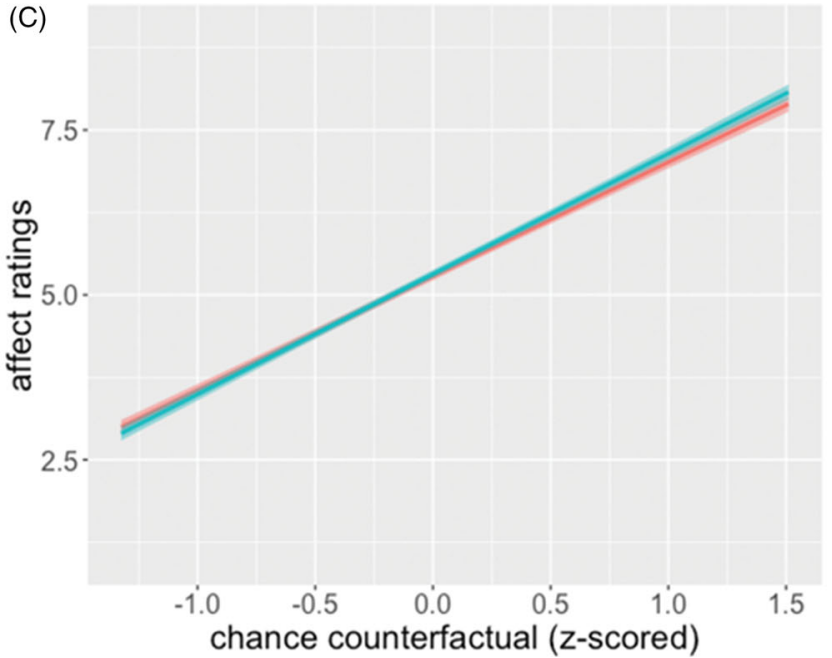

(B)

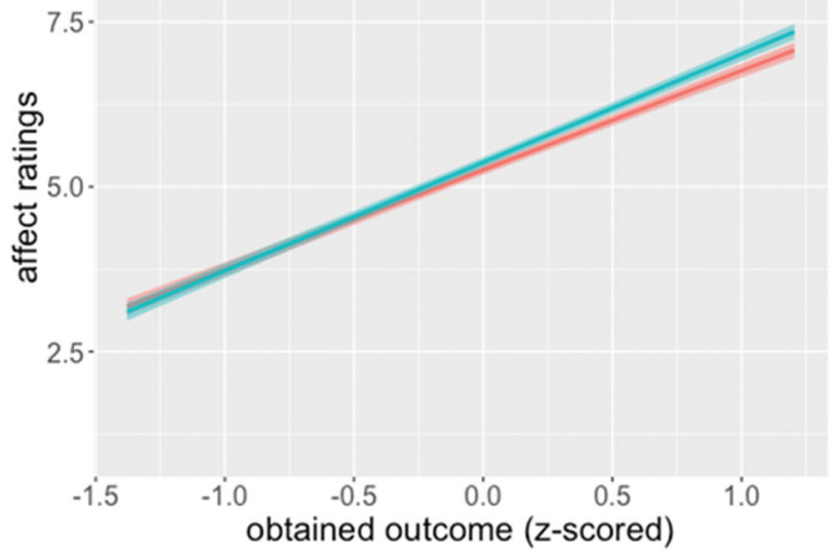

(D)

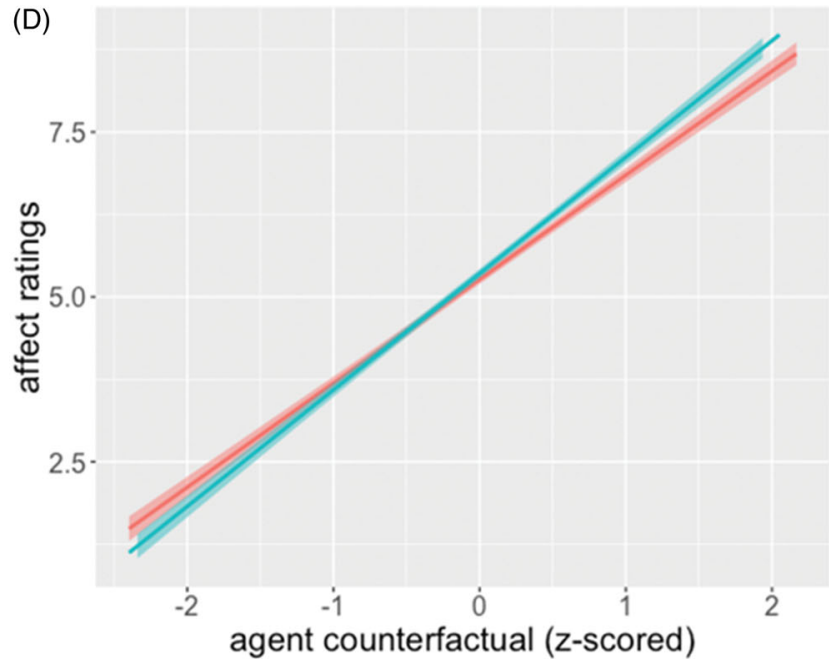

Figure 5. Affective ratings for partial and complete feedback. The left panels show the effect of obtained outcome (A) and chance counterfactual (B) affect rating following partial feedback. The right panels depict the effect of obtained outcome (C) and agent counterfactual (D) on affect rating following complete feedback. All the predictors are z-transformed. Shading around lines represent $95 \% \mathrm{Cl}$ for point estimates.

counterfactual and affect ratings was stronger in the stress condition $(b=1.76498, S E=0.03516, t=50.20, p<.001$, Cohen's $d=2.07)$ than in the control condition $(b=1.59218$, $S E=0.03499, t=45.50, p<.001$, Cohen's $d=1.87)$.

\subsection{Decision making}

3.5.1. Main effect of decision variable, expected value (e), risk variance $(v)$, and anticipated regret $(r)$

There was a significant main effect for e, whereby all participants exhibited a strong tendency to choose wheels based on anticipated economic utility (see Table 2). There was a significant main effect of $v$ such that all participants showed moderate risk-seeking tendencies, irrespective of the experimental condition. The main effect of $r$ was also significant, consistent with previous literature showing that individuals make choices as to minimize the likelihood of experiencing regret.

\subsubsection{Effects of stress on the choice model}

We tested for differences between stress and control conditions in the degree to which expected value $(e)$, risk variance
Table 2. Model of choice behavior using binary logistic regression.

\begin{tabular}{|c|c|c|c|c|c|}
\hline Parameter & Coefficient & Standard Error & z Value & $p$ Value & Cohen's $d$ \\
\hline \multicolumn{6}{|c|}{ (A) Choice Model with All subjects } \\
\hline Intercept & -0.063876 & 0.044980 & -1.42 & .16 & \\
\hline e & 0.821741 & 0.074596 & 11.016 & $<.001$ & 0.36 \\
\hline $\mathrm{v}$ & -0.347487 & 0.052860 & -6.574 & $<.001$ & 0.17 \\
\hline $\mathrm{r}$ & 0.929050 & 0.054499 & 17.047 & $<.001$ & 0.62 \\
\hline $\mathrm{e}^{*}$ treatment & -0.008438 & 0.105971 & -0.080 & .9365 & 0.02 \\
\hline $\mathrm{v}^{*}$ treatment & 0.002333 & 0.075980 & 0.031 & .9755 & 0.01 \\
\hline$r^{*}$ treatment & 0.197603 & 0.079278 & 2.493 & .0127 & 0.07 \\
\hline \multicolumn{6}{|c|}{60 participants, 4775 observations. Log Likelihood: -2477.7} \\
\hline \multicolumn{6}{|c|}{ (B) Choice Model in the stress condition } \\
\hline Intercept & 0.01263 & 0.07322 & 0.172 & .863 & \\
\hline e & 0.82752 & 0.07639 & 10.832 & $<.001$ & 0.49 \\
\hline $\mathrm{v}$ & -0.3 & & -6.343 & $<.001$ & 0.22 \\
\hline$r$ & 1.13139 & 0.05833 & 19.395 & $<.001$ & 1.04 \\
\hline \multirow{2}{*}{\multicolumn{6}{|c|}{30 participants, 2391 observations. Log Likelihood: -1204.5 }} \\
\hline & & & & & \\
\hline Intercept & -0.13619 & 0.05021 & -2.713 & $<.0$ & \\
\hline e & 0.80876 & 0.07429 & 10.886 & $<.001$ & 0.51 \\
\hline v & -0.34504 & 0.05272 & -6.544 & $<.001$ & 0.23 \\
\hline$r$ & 0.92593 & 0.05450 & 16.990 & $<.001$ & 0.86 \\
\hline
\end{tabular}

Panel $A$ shows results from the choice model containing parameters expected value, risk variance, and anticipation of regret and their interactions with group. Each coefficient in the full-choice model refers to the change in log odds per unit change in the given predictor. Panels $B$ and $C$ show results from applying the model to choice behavior of the stress and control groups separately. 

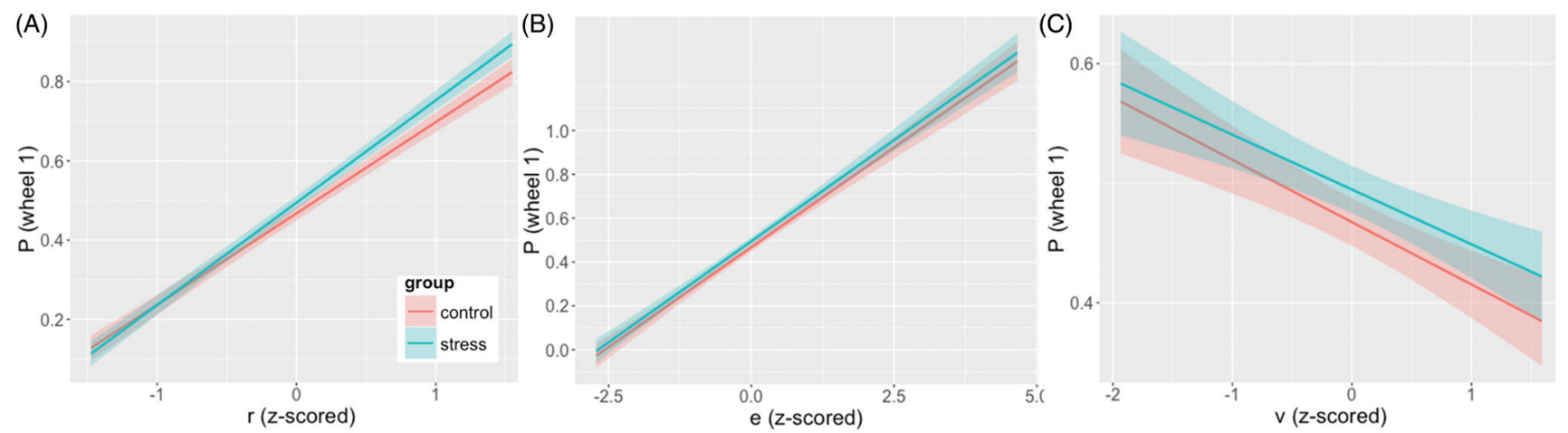

Figure 6. Behavioral sensitivity to regret, expected value, and risk variance. Panels illustrate the logit model-predicted probability of choosing wheel 1 at varying levels of anticipated regret (A), expected value (B) and risk variance (C). All the predictors are z-transformed. Shading around lines represent $95 \%$ Cl for point estimates.

$(v)$, and anticipated regret $(r)$ predicted choice behavior. To summarize the main findings, compared with the control group, the stress group was associated with an elevation in the anticipated regret parameter (see Figure 6). The two groups did not differ in expected value and risk variance parameters.

\section{Discussion}

By combining the Socially Evaluated Cold Pressor Test with a counterfactual decision-making task, the present study effectively increased subjective stress level and cortisol reactivity (Schwabe \& Schächinger, 2018) and showed that the acute stress induction influenced both how people reacted to their decision outcomes and the decisions they made. By using a computational approach, we were able to uncover how the three parameters, i.e. anticipated regret, risk variance and expected value, influence the underlying decision-making process, in addition to the affective responses as revealed by traditional regression analysis.

As for the reactions to the outcomes, the findings showed that participants responded more negatively when the obtained outcome was lower than the non-obtained outcome on the alternative wheel. Importantly, acute stress further amplified the sensitivity to the experienced regret. With regard to the decisions participants made, the result again revealed a stress-regret link. At the choice stage, computational modeling revealed that under stress, individuals were more likely to make regret-averse choices; that appeared to be made to avoid potential regret associated with the selected wheel. Taken together, these data demonstrate that acute stress amplifies both experienced and anticipated regret in the counterfactual decision-making.

Although the current study was not designed to test its predictive value, the findings we obtained can be meaningfully related to the more general dual process model of decision making. The model assumes that human decisionmaking can be characterized as resulting from two competing systems; one characterized as a rapid, automatic, emotional-based, intuitive process (i.e. System 1), and the other characterized as a slow, effortful, deliberate process (i.e. System 2) (Evans, 2003). Recent research on decision making under stress suggests that stress shifts the balance between the two systems such that it elicits a switch from an analytic reasoning system to more intuitive processes (Yu, 2016). For instance, an early study by Porcelli and Delgado (2009) showed that the decision bias of gain-loss asymmetry (i.e. risk-averse in the gain frame and risk-seeking in the loss frame) was further increased under stress, suggesting that stress makes individuals respond more on automatic decision biases. More recent research showed that exogenously administered cortisol impairs cognitive reflection and potentiates a shift from deliberate to intuitive processing in the cognitive reflection task, a well-established paradigm designed to capture individual's tendency to supress intuitive, automatic and incorrect responses in favor of deliberate thinking (Margittai et al., 2016). The current study adds to this literature by showing that stress may also elicit emotional decision-making by increasing the importance of regret. In this respect it is also interesting to connect our results to the observations that the experience of regret tends to be accompanied by increased physiological reactivity. For example, skin conductance response, an index of emotional arousal, was increased when viewing a better, non-obtained outcome on the rejected gamble (Camille et al., 2004). High non-obtained gains (regret) heightened facial muscle activity on the corrugator, which is highly responsive to negative emotional stimuli (Wu \& Clark, 2015). While these studies interpreted these measures as being a consequence of regret, the current findings suggest that (externally induced) stress may also amplify both experienced and anticipated regret.

Future research may benefit from further exploration of the neuropsychological underpinnings of regret. Previous research has identified neural mechanisms involved in the processing of regret. In particular, individuals were increasingly regret aversive, a cumulative effect reflected in enhanced activity within the medial orbitofrontal cortex and amygdala, key brain regions associated with emotional processing (Coricelli et al., 2007). Moreover, this pattern of brain activity that was associated with the experience of regret was also expressed before choice selection, indicating this same neural circuitry mediates both experience and anticipation of regret (Coricelli et al., 2005). It is well established that acute stress potentiates amygdala reactivity, the key brain structure for threat detection, vigilance regulation, and facilitation of memory for arousing experiences (see Roozendaal 
et al., 2009 for a review). Informed by these insights, one might expect that the stress-induced sensitivity to regret could be mediated by the enhanced amygdala response.

Future research could also benefit from following up on our findings that were not related to regret. In the present study, participants in the stress condition did not differ from those in the control condition in making choices to maximize their earnings based on the expected value. Moreover, both groups did not differ in their (moderate) risk-taking tendencies. That induced stress did not affect people's tendency to take risk may be relevant for the literature in this domain as well. Past research on risky decision-making under stress has yielded inconsistent results. While some studies did report laboratory-induced stress lead to more risk taking behavior (Preston et al., 2007; Putman et al., 2010; Starcke et al., 2016), others showed some negative or null findings (von Dawans et al., 2012), and these inconsistencies could be due to task features (i.e. risk vs. ambiguity), gender differences (i.e. men vs. women) or experimental frames (i.e. gain vs. loss). Future research delineating the effects of stress on risk preference could fruitfully benefit from econometrics modeling, i.e. utility and probability weighting functions underlying risk taking (Kandasamy et al., 2014). Recent research showed that an important factor that may influence individual differences in risk-taking under stress is psychiatric symptom severity, i.e. pathological anxiety (Charpentier et al., 2017), repetitive negative thinking (Stamatis et al., 2020). We would encourage further work taking this measure when investigating the effects of stress on decision-making.

Some issues warrant further discussion. First, we followed the standard SECPT and our sample size $(n=60$, both men and women) is comparable with previous studies using this protocol (Schwabe \& Schächinger, 2018). Cortisol responses were not affected by gender differences in our data. Nevertheless, we caution against drawing preliminary conclusions that gender differences would not be relevant in stress response and decision-making. Given the well-established gender differences in cortisol reactivity and emotional responses to stressor (Kirschbaum et al., 1999), future studies need to use a larger sample to test if acute stress affects emotion decision-making differentially in men and women. Second, it is well established that acute stress and chronic stress have differential effects upon physiological response and decision-making process, with chronic stress nullifying physiological reactivity, i.e. heart rate variability (Landolt et al., 2017). Future research could fruitfully investigate how chronic stress or stress-related disorders affect counterfactual decisions. Third, we only tested university students which, as previous research has indicated, may be vulnerable to stressors (e.g. Kuhnell et al., 2020). Future research needs to corroborate if the present findings also generalize to more diverse samples, which could contribute to an evidence base for targeted intervention. Fourth, the reliability of the BIS $(\alpha=0.52)$ was low and some of the reported effect sizes were small. Interpretation of these measures should be treated with caution. Future research could use more power experimental design to address this issue.

To summarize, by combining the SECPT with a counterfactual decision-making task, our data showed that acute stress amplifies both experience and anticipation of regret. These findings are consistent with the proposition that acute stress shifts the balance of dual process system and tilts decisionmaking toward more emotional and intuitive processing.

\section{Notes}

1. 4-point scale $(1=$ not at all, $4=$ very much so $)$ with 20 items in total, scores range between 20 and 80, Cronbach's $\alpha=0.71$ in the present sample.

2. 4 -point scale ( $1=$ rarely/never, $4=$ almost always/always $)$ with 30 items in total, scores range between 30 and 120 , Cronbach's $\alpha=0.52$.

3. 5 -point scale $(0=$ not true at all, $4=$ true nearly all of the time) with 25 items in total, scores range between 0 and 100 , Cronbach's $\alpha=0.91$.

4. In the stress group, we tested the gender difference on self-reported measures and cortisol reactivity. Gender had no significant effects on self-reported pain $(F(1,28)=0.01, p>.1)$, perceived stress $(F(1,28)$ $=0.27, p>.1)$ or self-reported anger $(F(1,28)=0.09, p>.1)$. Men and women did not differ on the AUC measures of saliva cortisol, $t$ (28) $=1.53, p>.1$.

5. The threshold for small effect size is $0.20 ; 0.50$ for medium effect size; 0.80 for large effect size.

\section{Acknowledgments}

We thank Yizhi Cheng and Jianxin Ou for their help with this study.

\section{Disclosure statement}

No potential conflict of interest was reported by the author(s).

\section{Funding}

This work was supported by the National Natural Science Foundation of China [31600923, 31872784], Guangdong Educational Science Research Fund [2018GXJK150], Shenzhen University Research Fund [SZUGS2020JG07, 860/000002110601] and the Shenzhen Peacock Plan [827-000233] to YW. The funding sources had no further role in the study design, data collection, analysis, interpretation, and decision to submit this manuscript for publication.

\section{Notes on contributor}

Yin Wu is assistant professor of psychology at Shenzhen University, China. He is interested in neuroendocrinology, judgment and decision making.

Eric van Dijk is chair of the Social, Economic and Organizational Psychology unit, Leiden University. His research concentrates on the understanding of economic and social decision making.

Hong Li is chair professor at Shenzhen University, China. He works on cognitive development and cognitive neuroscience.

YW and HL designed the study. YW conducted data collection, analyzed the data and wrote the paper. EvD provided critical revisions. All authors have approved the final version for submission.

\section{References}

Barr, D. J., Levy, R., Scheepers, C., \& Tily, H. J. (2013). Random effects structure for confirmatory hypothesis testing: Keep it maximal. Journal of Memory and Language, 68(3), 255-278. https://doi.org/10.1016/j.jml. 2012.11.001 
Barratt, E. S., Monahan, J., \& Steadman, H. J. (1994). Impulsiveness and aggression. Violence and Mental Disorder: Developments in Risk Assess, 10, 61-79.

Baskin-Sommers, A., Stuppy-Sullivan, A. M., \& Buckholtz, J. W. (2016). Psychopathic individuals exhibit but do not avoid regret during counterfactual decision making. Proceedings of the National Academy of Sciences of the United States of America, 113(50), 14438-14443. https:// doi.org/10.1073/pnas.1609985113

Bates, D., Maechler, M., \& Bolker, B. (2012). Ime4: Linear mixed-effects models using $\mathrm{S} 4$ classes.

Bishop, S. J., \& Gagne, C. (2018). Anxiety, depression, and decision making: A computational perspective. Annual Review of Neuroscience, 41, 371-388. https://doi.org/10.1146/annurev-neuro-080317-062007

Camille, N., Coricelli, G., Sallet, J., Pradat-Diehl, P., Duhamel, J.-R., \& Sirigu, A. (2004). The involvement of the orbitofrontal cortex in the experience of regret. Science, 304(5674), 1167-1170. https://doi.org/10.1126/ science. 1094550

Charpentier, C. J., Aylward, J., Roiser, J. P., \& Robinson, O. J. (2017). Enhanced risk aversion, but not loss aversion, in unmedicated pathological anxiety. Biological Psychiatry, 81(12), 1014-1022. https://doi.org/ 10.1016/j.biopsych.2016.12.010

Charpentier, C. J., De Martino, B., Sim, A. L., Sharot, T., \& Roiser, J. P. (2016). Emotion-induced loss aversion and striatal-amygdala coupling in low-anxious individuals. Social Cognitive and Affective Neuroscience, 11(4), 569-579. https://doi.org/10.1093/scan/nsv139

Connor, K. M., \& Davidson, J. R. T. (2003). Development of a new resilience scale: The Connor-Davidson Resilience Scale (CD-RISC). Depression and Anxiety, 18(2), 76-82. ). https://doi.org/10.1002/da. 10113

Coricelli, G., Critchley, H. D., Joffily, M., O'Doherty, J. P., Sirigu, A., \& Dolan, R. J. (2005). Regret and its avoidance: A neuroimaging study of choice behavior. Nature Neuroscience, 8(9), 1255-1262. https://doi.org/ 10.1038/nn1514

Coricelli, G., Dolan, R. J., \& Sirigu, A. (2007). Brain, emotion and decision making: The paradigmatic example of regret. Trends in Cognitive Sciences, 11(6), 258-265. https://doi.org/10.1016/j.tics.2007.04.003

Dickerson, S. S., \& Kemeny, M. E. (2004). Acute stressors and cortisol responses: A theoretical integration and synthesis of laboratory research. Psychological Bulletin, 130(3), 355-391. https://doi.org/10. 1037/0033-2909.130.3.355

Diller, J. W., Patros, C. H. G., \& Prentice, P. R. (2011). Temporal discounting and heart rate reactivity to stress. Behavioural Processes, 87(3), 306-309. https://doi.org/10.1016/j.beproc.2011.05.001

Domes, G., \& Zimmer, P. (2019). Acute stress enhances the sensitivity for facial emotions: A signal detection approach. Stress (Amsterdam, Netherlands), 22(4), 455-460. https://doi.org/10.1080/10253890.2019. 1593366

Engelmann, J. B., Berns, G. S., \& Dunlop, B. W. (2017). Hyper-responsivity to losses in the anterior insula during economic choice scales with depression severity. Psychological Medicine, 47(16), 2879-2891. https:// doi.org/10.1017/S0033291717001428

Evans, J. S. B. T. (2003). In two minds: Dual-process accounts of reasoning. Trends in Cognitive Sciences, 7(10), 454-459. https://doi.org/10. 1016/j.tics.2003.08.012

Gao, W., Stalder, T., Foley, P., Rauh, M., Deng, H., \& Kirschbaum, C. (2013). Quantitative analysis of steroid hormones in human hair using a column-switching LC-APCI-MS/MS assay. Journal of Chromatography. $B$, Analytical Technologies in the Biomedical and Life Sciences, 928, 1-8. https://doi.org/10.1016/j.jchromb.2013.03.008

Gillan, C. M., Morein-Zamir, S., Kaser, M., Fineberg, N. a., Sule, A., Sahakian, B. J., Cardinal, R. N., \& Robbins, T. W. (2014). Counterfactual processing of economic action-outcome alternatives in obsessive-compulsive disorder: Further evidence of impaired goal-directed behavior. Biological Psychiatry, 75(8), 639-646. https://doi.org/10.1016/j.biopsych. 2013.01.018

Kandasamy, N., Hardy, B., Page, L., Schaffner, M., Graggaber, J., Powlson, A. S., Fletcher, P. C., Gurnell, M., \& Coates, J. (2014). Cortisol shifts financial risk preferences. Proceedings of the National Academy of Sciences of the United States of America, 111(9), 3608-3613. https://doi. org/10.1073/pnas.1317908111
Kirschbaum, C., Kudielka, B. M., Gaab, J., Schommer, N. C., \& Hellhammer, D. H. (1999). Impact of gender, menstrual cycle phase, and oral contraceptives on the activity of the hypothalamus-pituitary-adrenal axis. Psychosomatic Medicine, 61(2), 154-162. https://doi.org/10.1097/ 00006842-199903000-00006

Kishida, K. T., Saez, I., Lohrenz, T., Witcher, M. R., Laxton, A. W., Tatter, S. B., White, J. P., Ellis, T. L., Phillips, P. E. M., \& Montague, P. R. (2016). Subsecond dopamine fluctuations in human striatum encode superposed error signals about actual and counterfactual reward. Proceedings of the National Academy of Sciences of the United States of America, 113(1), 200-205. https://doi.org/10.1073/pnas.1513619112

Kuhnell, R., Whitwell, Z., Arnold, S., Kingsley, M. I. C., Hale, M. W., Wahrendorf, M., Dragano, N., \& Wright, B. J. (2020). Assessing the association of university stress and physiological reactivity with decisionmaking among students. Stress, 23(2), 136-143. https://doi.org/10. 1080/10253890.2019.1651285

Landolt, K., Maruff, P., Horan, B., Kingsley, M., Kinsella, G., O'Halloran, P. D., Hale, M. W., \& Wright, B. J. (2017). Chronic work stress and decreased vagal tone impairs decision making and reaction time in jockeys. Psychoneuroendocrinology, 84, 151-158. https://doi.org/10. 1016/j.psyneuen.2017.07.238

Margittai, Z., Nave, G., Strombach, T., van Wingerden, M., Schwabe, L., \& Kalenscher, T. (2016). Exogenous cortisol causes a shift from deliberative to intuitive thinking. Psychoneuroendocrinology, 64, 131-135. https://doi.org/10.1016/j.psyneuen.2015.11.018

Mellers, B., Schwartz, A., \& Ritov, I. (1999). Emotion-based choice. Journal of Experimental Psychology: General, 128(3), 332-345. https://doi.org/ 10.1037/0096-3445.128.3.332

Montague, P. R., Dolan, R. J., Friston, K. J., \& Dayan, P. (2012). Computational psychiatry. Trends in Cognitive Sciences, 16(1), 72-80. https://doi.org/10.1016/j.tics.2011.11.018

Pessoa, L., \& Adolphs, R. (2010). Emotion processing and the amygdala: From a 'low road' to 'many roads' of evaluating biological significance. Nature Reviews. Neuroscience, 11(11), 773-782. https://doi.org/10.1038/ nrn2920

Porcelli, A. J., \& Delgado, M. R. (2009). Acute stress modulates risk taking in financial decision making. Psychological Science, 20(3), 278-283. https://doi.org/10.1111/j.1467-9280.2009.02288.x

Preston, S. D., Buchanan, T. W., Stansfield, R. B., \& Bechara, a. (2007). Effects of anticipatory stress on decision making in a gambling task. Behavioral Neuroscience, 121(2), 257-263. https://doi.org/10.1037/07357044.121.2.257

Pruessner, J. C., Kirschbaum, C., Meinlschmid, G., \& Hellhammer, D. H. (2003). Two formulas for computation of the area under the curve represent measures of total hormone concentration versus timedependent change. Psychoneuroendocrinology, 28(7), 916-931. https:// doi.org/10.1016/S0306-4530(02)00108-7

Putman, P., Antypa, N., Crysovergi, P., \& van der Does, W. A. J. (2010). Exogenous cortisol acutely influences motivated decision making in healthy young men. Psychopharmacology, 208(2), 257-263. https://doi. org/10.1007/s00213-009-1725-y

Raio, C. M., Hartley, C. A., Orederu, T. A., Li, J., \& Phelps, E. A. (2017). Stress attenuates the flexible updating of aversive value. Proceedings of the National Academy of Sciences, 114(42), 11241-11246. https://doi. org/10.1073/pnas.1702565114

Robinson, O. J., Bond, R. L., \& Roiser, J. P. (2015). The impact of stress on financial decision-making varies as a function of depression and anxiety symptoms. PeerJ, 3, e770. https://doi.org/10.7717/peerj.770

Roozendaal, B., McEwen, B. S., \& Chattarji, S. (2009). Stress, memory and the amygdala. Nature Reviews. Neuroscience, 10(6), 423-433. https:// doi.org/10.1038/nrn2651

Schwabe, L., Haddad, L., \& Schachinger, H. (2008). HPA axis activation by a socially evaluated cold-pressor test. Psychoneuroendocrinology, 33(6), 890-895. https://doi.org/10.1016/j.psyneuen.2008.03.001

Schwabe, L., \& Schächinger, H. (2018). Ten years of research with the Socially Evaluated Cold Pressor Test: Data from the past and guidelines for the future. Psychoneuroendocrinology, 92, 155-161. https:// doi.org/10.1016/j.psyneuen.2018.03.010 
Speer, M. E., \& Delgado, M. R. (2017). Reminiscing about positive memories buffers acute stress responses. Nature Human Behaviour, 1, 0093. https://doi.org/10.1038/s41562-017-0093

Spielberger, C. D., Gorsuch, R. L., Lushene, R., Vagg, P. R., \& Jacobs, G. A. (1983). Manual for the state-trait anxiety scale. Palo Alto, CA: Consulting Psychology Press.

Stamatis, C. A., Puccetti, N. A., Charpentier, C. J., Heller, A. S., \& Timpano, K. R. (2020). Repetitive negative thinking following exposure to a natural stressor prospectively predicts altered stress responding and decision-making in the laboratory. Behaviour Research and Therapy, 129, 103609. https://doi.org/10.1016/j.brat.2020.103609

Starcke, K., \& Brand, M. (2012). Decision making under stress: A selective review. Neuroscience and Biobehavioral Reviews, 36(4), 1228-1248. https://doi.org/10.1016/j.neubiorev.2012.02.003

Starcke, K., Brand, M., \& Kluge, A. (2016). Stress influences decisions to break a safety rule in a complex simulation task in females. Biological Psychology, 118, 35-43. https://doi.org/10.1016/j.biopsycho.2016.04.067

Trapp, S., O'Doherty, J. P., \& Schwabe, L. (2018). Stressful events as teaching signals for the brain. Trends in Cognitive Sciences, 22(6), 475-478. https://doi.org/10.1016/j.tics.2018.03.007

Van Dijk, E., \& Zeelenberg, M. (2005). On the psychology of 'if only': Regret and the comparison between factual and counterfactual outcomes. Organizational Behavior and Human Decision Processes, 97(2), 152-160. https://doi.org/10.1016/j.obhdp.2005.04.001 von Dawans, B., Fischbacher, U., Kirschbaum, C., Fehr, E., \& Heinrichs, M. (2012). The social dimension of stress reactivity. Psychological Science, 23(6), 651-660. https://doi.org/10.1177/0956797611431576

Wieser, M. J., Pauli, P., Reicherts, P., \& Mühlberger, A. (2010). Don't look at me in anger! Enhanced processing of angry faces in anticipation of public speaking. Psychophysiology, 47(2), 271-280. https://doi.org/10. 1111/j.1469-8986.2009.00938.x

Wolf, O. T., Schulte, J. M., Drimalla, H., Hamacher-Dang, T. C., Knoch, D., \& Dziobek, I. (2015). Enhanced emotional empathy after psychosocial stress in young healthy men. Stress, 18(6), 631-637. https://doi.org/10. 3109/10253890.2015.1078787

Wu, Y., \& Clark, L. (2015). Disappointment and regret enhance corrugator reactivity in a gambling task. Psychophysiology, 52(4), 518-523. https:// doi.org/10.1111/psyp.12371

Wu, Y., Clark, L., Zilioli, S., Eisenegger, C., Gillan, C. M., Deng, H., \& Li, H. (2018). Single dose testosterone administration modulates emotional reactivity and counterfactual choice in healthy males. Psychoneuroendocrinology, 90, 127-133. https://doi.org/10.1016/j.psyneuen.2018.02.018

$\mathrm{Yu}, \mathrm{R}$. (2016). Stress potentiates decision biases: A stress induced deliberation-to-intuition (SIDI) model. Neurobiology of Stress, 3, 83-95. https:// doi.org/10.1016/j.ynstr.2015.12.006

Zeelenberg, M. (1999). Anticipated regret, expected feedback and behavioral decision making. Journal of Behavioral Decision Making, 12(2), 93-106. https://doi.org/10.1002/(SICI)1099-0771(199906)12:2<93::AID$\mathrm{BDM} 311>3.0 . \mathrm{CO} ; 2-\mathrm{S}$ 